\title{
The Outcome of Infants and Children with Heart Muscle Disease: Prognosis in Centers without Heart Transplantation Option
}

\author{
L. Kampel1', Z. Perles' ${ }^{2}$ A. J. J. T. Rein², N. El-Lahham², J. Braun², \\ S. Gavri², J. Golender' ${ }^{2}$ A. Nir ${ }^{2 *}$ \\ ${ }^{1}$ Aerospace Medical Unit, the Israeli Air Force, Tel Hashomer, Israel \\ ${ }^{2}$ Hadassah-Hebrew University Medical Center, Jerusalem, Israel \\ Email: amiramn@ekmd.huji.ac.il
}

Received 29 December 2013; revised 25 January 2014; accepted 3 February 2014

Copyright (C) 2014 by authors and Scientific Research Publishing Inc.

This work is licensed under the Creative Commons Attribution International License (CC BY). http://creativecommons.org/licenses/by/4.0/

(c) (i) Open Access

\begin{abstract}
Objective: Acute heart failure due to heart muscle disease is potentially fatal in pediatric patients. Heart transplantation (Tx) is utilized to improve survival. However, spontaneous recovery may occur. The outcome of patients treated in centers with no Tx option was studied. Patients and Methods: A retrospective review of infants and children ( $\leq 16$ years) who presented with heart muscle disease (left ventricular shortening fraction, LVSF $\leq 25 \%$ ) between 1992 and 2007, in two medical centers in Jerusalem, was performed. Results: There were 62 patients, mean age 2.5 years, mean LVSF $16.3 \%$. The 5 -year survival was $73 \%$. Lower LVSF at presentation $(p=0.006)$ was independently associated with mortality. Recovery (normalization of LVSF) occurred in $56 \%$ of survivors. of the 38 patients who were eligible for Tx at their presentation, according to the AHA guidelines, $22(58 \%)$ survived and $13(34 \%)$ recovered. Conclusions: This study shows the long term outcome of pediatric patients with acute heart muscle disease treated with no Tx option. These findings may reflect the impact of improved medical management of these patients in recent years.
\end{abstract}

\section{Keywords}

Pediatrics; Cardiomyopathy; Heart Failure; Survival; Transplantation

\section{Introduction}

Pediatric patients with cardiomyopathies, presenting with acute heart failure, are known to have high mortality

"Corresponding author.

How to cite this paper: Kampel, L., et al. (2014) The Outcome of Infants and Children with Heart Muscle Disease: Prognosis in Centers without Heart Transplantation Option. Open Journal of Pediatrics, 4, 34-41. 
[1]. However, despite their severe presentation, survival and spontaneous recovery of left ventricular function are possible [2]-[4]. As there are no known factors that could accurately determine who is at risk for death [5] [6], the natural history of acute heart failure due to cardiomyopathy is still unpredictable. Therapy for this condition includes medications, mechanical support and heart transplantation. The latter is an irreversible procedure. It is believed that heart transplantation improves the survival for selected patients [6] [7]. The American Heart Association (AHA) published practical guidelines for heart transplantation in pediatric patients with heart failure associated with cardiomyopathies [8]. Yet, the decision to proceed to transplantation is complex, as at the time a donor heart becomes available, the risk for death is hard to determine, and some patients may undergo transplantation despite having a curable disease.

As most reports combine death and heart transplantation as a measure of cardiac decompensation, the proportion of patients who would have survived without heart transplantation is unknown. These data are important since heart transplantation is associated with significant morbidity and mortality. Available historical figures may not reflect the survival with the current medical and intensive care therapy.

The present study reviews the course of pediatric patients with heart failure due to heart muscle disease, in two medical centers, where no pediatric heart transplantation was performed during the years of the study. The results reflect the contribution of modern medications in clinical practice to the outcome of pediatric cardiomyopathy.

\section{Methods}

\subsection{Patients and Data Collection}

Data was obtained from two university medical centers: Hadassah University Hospital and Shaare Zedek Medical Center. The study population was confined to infants and children younger than 16 years of age, diagnosed with heart muscle disease, between Jan 1, 1992 and Dec 31, 2007. Patients included were diagnosed with cardiomyopathy (based on the attending cardiologist consultation) and had an echocardiographic evidence of left ventricular dysfunction, defined as left ventricular shortening fraction (LVSF) $\leq 25 \%$. In the medical centers of this study, endomyocardial biopsy is not routinely performed, thus there was no distinction between myocarditis and dilated cardiomyopathy. Elective cardiac biopsy was recorded when carried out. Patients with structural congenital heart disease, metabolic disorders, ischemic injury, progressive neuromuscular disease, primary pulmonary hypertension and septicemia were excluded.

Demographic and clinical data at presentation were retrospectively collected. Personal or familial history of cardiomyopathy was noted, as well as a history of preceding viral illness within two weeks before admission (acute fever, respiratory tract infection or gastrointestinal infection). Medications administered during hospitalization, intensive care unit (ICU) admission and length of stay in ICU were also recorded. Mortality data was obtained from patients files and computerized systems synchronized with the inhabitants' registry.

Echocardiographic variables consisting of left ventricular end-diastolic diameter (LVEDD), left ventricular end-systolic diameter (LVESD) and left ventricular shortening fraction (LVSF), were recorded during hospitalization, and at follow up examinations. In order to overcome the age- and growth-related alternations in myocardial mechanics [9], adjusted variables were used: an age-adjusted parameter of fractional shortening: LVSF/ Lower Normal Limit of LVSF according to age (\%) and BSA-adjusted LVEDD (LVEDD 95\%).

Among the study group, patients who were possible heart transplantation candidates according to the AHA guidelines [8], were identified. In the guidelines, Class I recommendation indicates heart transplantation as therapy for stage D heart failure associated with systemic ventricular dysfunction in pediatric patients with cardiomyopathies. Stage D is defined as abnormal cardiac structure and/or function, with continuous infusion of intravenous inotropes and/or mechanical ventilatory or mechanical circulatory support.

\subsection{Statistical Methods}

Outcome measures included death or survival. Within surviving group, two subgroups were defined, according to their last follow-up echocardiographic examination: patients with persistent cardiomyopathy (Myopathy), and patients who recovered (Recovery - defined by LVSF $\geq$ lower normal limit of LVSF according to age).

The two-tail t-test, as well as the non-parametric Mann-Whitney test, was applied in order to compare quantitative variables among patient outcomes. The Kruskal-Wallis non-parametric test was used to compare the 
quantitative variables among final three outcomes (Death, Myopathy and Recovery), since the number of patients in each group was small. Chi-square test was applied for the comparison of our results to published data. In order to test the association between patient categorical variables and outcome, the Chi-square test and the Fisher's exact test were performed. Based on the results of the univariate analyses, the logistic regression model was applied. A hazard ratio, with 95\% confidence interval, was generated for each covariate, which was predictive of outcome. Kaplan-Meier models were used to assess survival, using the log-rank test for the comparison of survival curves. All tests applied were two-tailed, and a p-value of 0.05 or less was considered as statistically significant.

\section{Results}

Sixty five patients met the inclusion criteria. Three of them were lost to follow up, thus excluded. Mean followup interval (i.e time from admission to the last examination recorded in the study medical centers) was 34 months.

\subsection{Clinical Presentation and Initial Therapy}

Table 1 summarizes the characteristics of the study population. The median age at presentation was 1.15 years (range, 0 - 14 years). The distribution of the study population according to age-groups is shown in Table 1 . The left ventricular shortening fraction ranged from $8 \%$ to $25 \%$ (median $16.75 \%)$. The majority $(n=48,77 \%)$ had LVSF $\leq 20 \%$. Congestive heart failure as presenting symptom was reported in 55 patients (89\%), 11 (18\%) presented with cardiogenic shock, 37 patients (60\%) were admitted to intensive care unit at first presentation. Three

\begin{tabular}{|c|c|}
\hline Variables & No of patients $(\%)$ \\
\hline \multicolumn{2}{|l|}{ Age at presentation: } \\
\hline$\leq 1$ month & $10(16.1)$ \\
\hline$>1$ month and $\leq 1$ year & $20(32.3)$ \\
\hline$>1$ year and $\leq 5$ years & $24(38.7)$ \\
\hline$>5$ years & $8(12.9)$ \\
\hline Female gender & $36(58.1)$ \\
\hline ICU admission at presentation & 37 (59.7) \\
\hline Preceding viral illness & $28(46.7)$ \\
\hline Family history of cardiomyopathy & $7(11.3)$ \\
\hline \multicolumn{2}{|c|}{ Medications within 1 week of presentation } \\
\hline Digoxin & $38(61.3)$ \\
\hline Diuretics & $41(66.1)$ \\
\hline ACE I & $40(64.5)$ \\
\hline Steroids & $23(37.1)$ \\
\hline IVIG & 19 (30.6) \\
\hline Inotrops (Dopamine, Dobutamine) & $33(53.2)$ \\
\hline Milrinone & $14(22.6)$ \\
\hline \multicolumn{2}{|l|}{ LVSF at presentation } \\
\hline$<15$ & $24(38.7)$ \\
\hline$\geq 15$ and $<20$ & $20(32.3)$ \\
\hline$\geq 20$ & $18(29)$ \\
\hline LVEDD $>$ 95\%tile $[n=48]^{*}$ & $28(58)$ \\
\hline
\end{tabular}

IVIG = Intravenous immunoglobulins; LVSF = Left Ventricular; Shortening Fraction; LVEDD = Left Ventricular End Diastolic Diameter; "This echocardiographic result was not available for some subjects. 
patients (5\%) presented with arrhythmia and 4 patients (6\%) were asymptomatic. Two of the 4 were diagnosed because of a heart murmur, and another two were diagnosed on screening of familial cardiomyopathy. Familial cardiomyopathy was identified in 7 patients (11\%). Other causes included 2 cases of left ventricular noncompaction cardiomyopathy and 1 antracycline-induced cardiomyopathy. Biopsy confirmed myocarditis was reported in 1 patient (parvovirus myocarditis) and another endomyocardial biopsy taken revealed lymphocytic infiltrates consistent with acute myocarditis. Overall, only these 2 biopsies, out of 8 performed, had diagnostic significance. Four patients had suggestive signs and inflammatory markers of myocarditis, with positive polymerase chain reaction for enterovirus, RSV, rotavirus and CMV. A history of viral illness before diagnosis was reported in 28 patients, 22 patients (35\%) had respiratory tract infection signs, 5 (8\%) had gastroenteritis and 1 had acute fever with rash. The medications delivered are presented in Table 1. Intravenous inotropic support was administered to 33 patients (53\%) on first admission. Twenty nine patients required mechanical ventilation. Since extracorporeal membrane oxygenation (ECMO) was not available in the study medical centers, only 3 patients were considered to be transported to centers where ECMO is used. None of them survived until ECMO was available. Heart transplantation was discussed in the medical records of 5 patients. Two children and 1 infant died before transplantation could be carried out. Another patient was transferred to a European transplantations center, but did not undergo transplantation since she was diagnosed with viral myocarditis based on an endomyocardial biopsy. The fifth patient was found to have secondary pulmonary hypertension, hence was not suitable for heart transplantation.

\subsection{Outcome}

Seventeen patients (27\%) died during the 15-years period of the study. Fifty two percent $(n=9)$ died within the first week of their first hospitalization. Actuarial survival is presented in the Kaplan-Meier survival curve (Figure 1(a)). The 5-year survival in this study group was 73\%. This result is not significantly different from the 5-year survival reported by tertiary care centers, utilizing pediatric heart transplantations, while their survival rates included the transplanted patients [1] [6] [10]-[15].

In the present study, at the last follow up, 25 patients (40\%) showed recovery (normalization of LV function) and 20 (32\%) had persistent cardiomyopathy. Recovery occurred mostly during the first year (88\% of patients, $36 \%$ recovered within one month); however the left ventricular function of 3 patients recovered more than a year after presentation.

\subsection{Factors Related to Outcome}

There was a difference in survival according to age-groups at presentation ( $\mathrm{p}=0.035$ ). Only $40 \%$ (4 out of 10 ) of patients aged 1 month or younger at presentation survived, whereas $75 \%$ of those presenting between 1 month and 1 year, and $87.5 \%$ between 1 to 5 years, survived. Children over 5 years of age had a survival rate of $62.5 \%$. Fifty percent of the patients younger than 1 month of age presented with severe acute myocardial failure, and were in cardiogenic shock. The survival of all patients older than 1 month was $79 \%$. The differences in survival according to age-groups are shown in Figure 1(b). Other factors associated with mortality were the use of intravenous inotropic support and lower left ventricular shortening fraction at presentation. Left ventricular shortening fraction was significantly lower in patients who died (mean $13.8 \%$ Vs $17.2 \%$ in survivors, $p=0.014$ ). For every unit percent lower fractional shortening, there was a 1.22 higher risk for death. No association was found between LVEDD or LVESD and outcome. Other factors with no significant correlation to specific outcome were gender, family history of cardiomyopathy, preceding viral illness, admission to ICU at presentation or the use of any cardiac medications. Factors found to be predictive for death in the multivariable proportional hazard model were fractional shortening and the use of inotropic support (Table 2).

\subsection{Characteristics and Outcome of Potential Heart-Transplant Candidates}

Thirty eight patients (61\%) were potential candidates for transplantation on admission to hospital, since they met stage D criteria. Figure 2 shows the number of patients suitable for transplantation over one month of hospitalization, categorized by their outcome according to the last follow-up examination. Recovery of left ventricular function (normalization of LVFS) was found in 34\% of patients who could potentially be transplanted on the first day of hospitalization, and $47 \%$ of patients — on the fifth day. Within the group of patients categorized as 


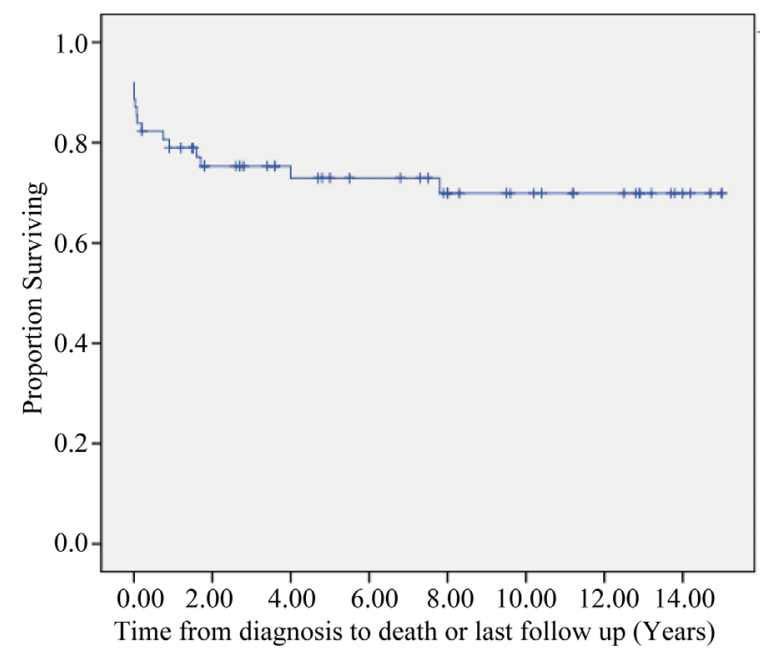

(a)

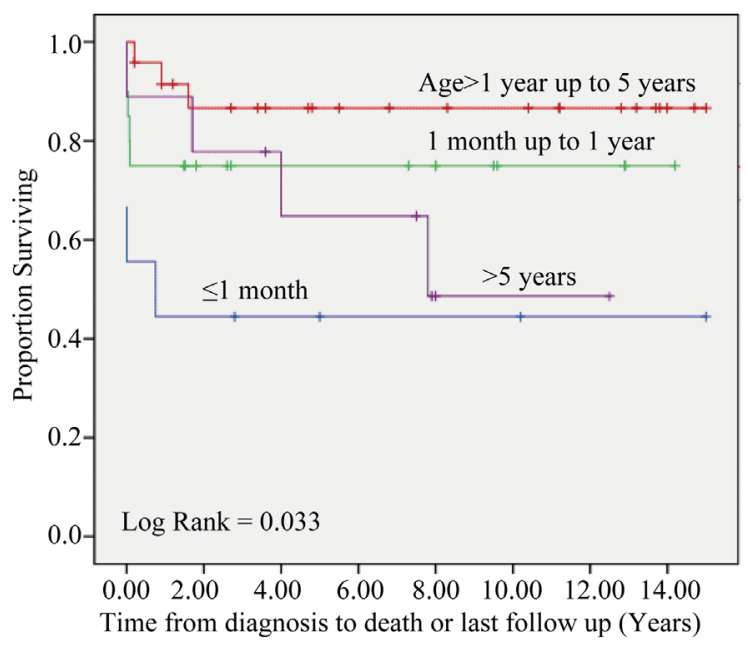

(b)

Figure 1. Survival of pediatric patients with cardiomyopathy. Kaplan Meier survival curve: (a) the entire group; (b) patients grouped by age at presentation. Last follow-up times are indicated by "+” for patients alive at the end of the study. A significant survival difference is observed between age groups $(\mathrm{p}<0.035)$.

Table 2. Factors at presentation related to death ${ }^{*}$.

\begin{tabular}{ccccc}
\hline & Hazard Ratio & 95\% CI & Significance \\
\hline Low LVSF & & 1.412 & 0.006 \\
Inotropic support & 1.22 & 1.060 & 24.4 & 0.025 \\
\hline
\end{tabular}

${ }^{*}$ Cox proportional hazards model; ${ }^{\dagger}$ Per unit - percent reduction in fractional shortening.

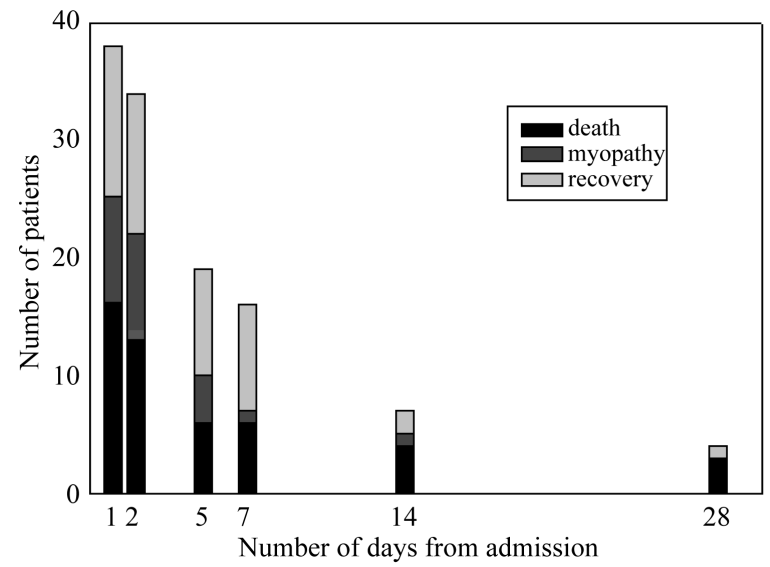

Figure 2. Outcome probabilities of patients eligible for heart transplantation. The final outcome (death, recovery or myopathy) of patients, eligible for heart transplantation, on different days from admission.

stage $\mathrm{D}$ on the day of admission, lower LVSF\% at presentation was associated with death $(\mathrm{p}=0.01)$. Among patients who still met stage $\mathrm{D}$ criteria on day 5 of hospitalization, the majority who recovered, were female $(\mathrm{p}=$ 0.01). However, the association between mortality and lower LVSF\% did not remain significant, in that group (Table 3).

\section{Discussion}

At present, cardiac transplantation provides the final alternative when medical treatment apparently fails in cases 
Table 3. Characteristics associated with specific outcomes in patients eligible for heart transplantation (stage D).

\begin{tabular}{|c|c|c|c|c|}
\hline & Recovery & Persistent Myopathy & Death & p Value \\
\hline \multicolumn{5}{|l|}{ Stage $D$ on day $1[\mathrm{n}=38]$} \\
\hline \multicolumn{5}{|l|}{ Gender } \\
\hline Female & $39 \%$ & $26 \%$ & $35 \%$ & $0.443^{*}$ \\
\hline Male & $20 \%$ & $27 \%$ & $53 \%$ & \\
\hline LVSF, \% (mean \pm SD) & $15.3 \pm 3.6$ & $16.7 \pm 3.1$ & $12.3 \pm 3.4$ & $0.01^{\dagger}$ \\
\hline LVEDD 95\% $($ mean \pm SD) & $105.9 \pm 15.4$ & $107.8 \pm 20.4$ & $107.2 \pm 28.3$ & $0.916^{\dagger}$ \\
\hline \multicolumn{5}{|l|}{ Stage $D$ on day $5[n=19]$} \\
\hline \multicolumn{5}{|l|}{ Gender } \\
\hline Female & $66 \%$ & $17 \%$ & $17 \%$ & $0.01^{*}$ \\
\hline Male & $0 \%$ & $43 \%$ & $57 \%$ & \\
\hline LVSF, \% (mean \pm SD) & $14 \pm 3.5$ & $15.2 \pm 2.4$ & $11.5 \pm 2.7$ & $0.096^{\dagger}$ \\
\hline LVEDD 95\% (mean \pm SD) & $106.6 \pm 18.8$ & $107.7 \pm 23.8$ & $118.6 \pm 30.7$ & $0.748^{\dagger}$ \\
\hline
\end{tabular}

${ }^{*}$ Chi Square test; ${ }^{\dagger}$ Kruskal Wallis test.

of acute heart failure due to heart muscle disease. The complexity of the decision to proceed with transplantation is attributable, in part, to the possibility of eventual improvement and resolution of left ventricular dysfunction [3] [4]. When comparing outcome of pediatric dilated cardiomyopathy with and without transplantation, studies of 20 - 30 years ago are used for outcome with no heart transplant option [16]. The present study provides prognostic information on pediatric DCM with modern medical management and no heart transplantation and relates to the heart transplantation eligibility as reflected by the guidelines. In a recent report of pediatric Heart transplant registry, of patients on the waiting list who were not transplanted, 52\% were alive and $48 \%$ dead at 2 years [17]. Similar figures are reported in 261 children with dilated cardiomyopathy [18]. In the present study, of patients whose clinical presentation was severe enough to fulfill class I recommendation for heart transplantation, according to the AHA guidelines ( $\mathrm{n}=38$ ), 58\% survived and $34 \%$ recovered. Amongst patients who were eligible for transplantation 5 days following admission $(n=19)$, the rate of eventual recovery was considerably high (47\%). These figures suggest that patients eligible for heart transplantation at early stages of the disease have about $50 \%$ chance for survival without transplantation. Our data further suggests that significant minority of these patients may recover. In centers with transplant option the mean time from diagnosis to listing is reported to be $0.62 \pm 1.3$ years [18], suggesting that most centres do not list patients for transplantation in the first days after admission.

The patients admitted with stage D heart failure, as their first presentation, give rise to critical questions regarding their management. The dilemma of whether to go ahead with transplant or wait is difficult [12]. The present study adds important information when facing a patient with deteriorating left ventricular function who requires intravenous or mechanical support, and a suitable heart becomes available. Early in the course of the disease both mortality and the potential for recovery are high and it is often hard to predict the prognosis. Furthermore, there is no absolutely reliable and accurate diagnostic test, for determining the underlying etiology. As the optimal timing for transplantation has not yet been defined, according to current transplantation guidelines, patients who might have recovered and lived on with their own heart-may be condemned to living with an implant, and the considerable morbidity and mortality it implies. The results of the present study suggest that there is a significant rate of survival and recovery without transplantation. Mechanical support was not available in the study period, suggesting that survival may have been even better than reported here. There is no doubt that many patients are saved by heart transplantation, but if one follows the current AHA recommendations, some can be transplanted early in their clinical course when they may have done better without transplantation. Recent data shows a 5-year survival of children with dilated cardiomyopathy after heart transplant of 83\% [17].

In the present study, significant independent risk factors for death, in patients with heart muscle diseases, included requirement of intravenous inotropic support and reduced left ventricular shortening fraction on the initial echocardiogram. Similar associations were reported by others as well [1] [5] [6] [10] [15]. The age at presentation may have a predictive value, as the youngest patients (less than 4 weeks of age) were less likely to sur- 
vive. The low number of patients in this age group $(n=10)$ limited the ability to achieve statistical significance, but this trend of poorer outcome according to similar age distribution has been reported [5].

Gender, family history of cardiomyopathy or a history of previous viral illness were found to have no indicative significance regarding any outcome. These findings are in agreement with other reports [2] [10] [11]. Evidently, as already indicated by others, a more comprehensive risk profile is required in order to provide better selection of care to pediatric patients with cardiomyopathy.

\section{Study Limitations}

The data in this study were retrospectively collected. As a result, there were considerable differences between patients' medical records. Clinical management did not follow a standardized protocol, and various considerations of the attending cardiologist at the time, led to the use or no-use of different medications.

It is possible that there is dissimilarity between the patients included in this study, that represent the population of Jerusalem and its rural areas, and the population of other studies, with which survival rates were compared. However, the degree of left ventricular dysfunction, as expressed by LVSF in our study, is similar to the reported in other studies. Another limitation of this study is the diverse etiological considerations. Since endomyocardial biopsy is not routinely undertaken in the study medical centers, the etiology of left ventricular dysfunction was not always well established. The risk of endomyocardial biopsy seems to outweigh its benefit in children [14] [15]. Thus, current practice does not necessitate cardiac biopsy in the acute clinical setup. As in many centers there is no definite etiological diagnosis to a patient with acute heart failure due to left ventricular dysfunction, the data presented here is relevant to real life situations.

Follow up was not optimal for all patients, since the population of the study represents both locals and referrals from other regions. The latter lacked frequent follow-up examinations, and had a limited follow-up interval.

\section{Conclusion}

This study shows that dilated cardiomyopathy in childhood carries significant morbidity and mortality. A significant proportion of patients who fulfill the criteria for heart transplantation may survive and even recover with medical therapy.

\section{Disclosures}

There are no conflicts of interest to disclose. There was no funding source for this study.

\section{References}

[1] Towbin, J.A., Lowe, A.M., Colan, S.D., Sleeper, L.A. and Lipshultz, S.E. (2006) Incidence, Causes and Outcomes of Dilated Cardiomyopathy in Children. JAMA, 286, 1867-1876. http://dx.doi.org/10.1001/jama.296.15.1867

[2] Arola, A., Tuominen, J., Ruskanen, O. and Jokinen, E. (1998) Idiopathic Dilated Cardiomyopathy in Children: Prognostic Indicators and Outcome. Pediatrics, 101, 369-376. http://dx.doi.org/10.1542/peds.101.3.369

[3] Lewis, A.B. (1999) Late Recovery of Ventricular Function in Children with Idiopathic Dilated Cardiomyopathy. American Heart Journal, 138, 334-338. http://dx.doi.org/10.1016/S0002-8703(99)70121-3

[4] O’Sullivan, J.J., Roche, S.L., Crossland, D.S., Chaudhari, M.P., Kirk, R.C. and Asif, H. (2008) Recovery of Heart Function in Children with Acute Severe Heart Failure. Transplantation, 85, 975-979. http://dx.doi.org/10.1097/TP.0b013e318168fe3c

[5] Daubeney, P.E.F., Nugent, A.W. and Chondoros, P. (2006) Clinical Features and Outcomes of Childhood Dilated Cardiomyopathy: Results from a National Population-Based Study. Circulation, 114, 2671-2678. http://dx.doi.org/10.1161/CIRCULATIONAHA.106.635128

[6] Tsirka, A.E., Trinkaus, K. and Chen, S. (2004) Improved Outcomes of Pediatric Dilated Cardiomyopathy with Utilization of Heart Transplant. Journal of the American College of Cardiology, 44, 391-397. http://dx.doi.org/10.1016/j.jacc.2004.04.035

[7] Canter, C.E. and Kantor, P.F. (2007) Heart Transplant for Pediatric Cardiomyopathy. Progress in Pediatric Cardiology, 23, 67-72. http://dx.doi.org/10.1016/j.ppedcard.2007.05.012

[8] Canter, C.E., Shaddy, R.E. and Bernstein, D. (2007) Indications for Heart Transplantation in Pediatric Heart Disease. Circulation, 115, 658-676. http://dx.doi.org/10.1161/CIRCULATIONAHA.106.180449 
[9] Colan, S.D., Parness, I.A., Spevak, P.J. and Sanders, S.P. (1992) Developmental Modulation of Myocardial Mechanics: Age- and Growth-Related Alternations in Afterload and Contractility. Journal of the American College of Cardiology, 19, 619-629. http://dx.doi.org/10.1016/S0735-1097(10)80282-7

[10] Azevedo, V.M.P., Santos, M.A., Albanesi Filho, F.M., Castier, M.B., Tura, B.R. and Amino, J.G.C. (2007) Outcome Factors of Idiopathic Dilated Cardiomyopathy in Children-A Long-Term Follow Up Review. Cardiology in the Young, 17, 175-184. http://dx.doi.org/10.1017/S1047951107000170

[11] Alvarez, J.A., Wilkinson, J.D. and Lipshultz, S.E. (2007) Outcome Predictors for Pediatric Dilated Cardiomyopathy: A Systematic Review. Progress in Pediatric Cardiology, 23, 25-32. http://dx.doi.org/10.1016/j.ppedcard.2007.05.009

[12] Goldmen, A.P. and Cassidy, J. (2003) The Waiting Game: Bridging to Pediatric Heart Transplantation. Lancet, 362, 1967-1970. http://dx.doi.org/10.1016/S0140-6736(03)15015-5

[13] Kirk, R., Edwards, L.B., Aurora, P., Taylor, D.O. and Hertz, M.I. (2008) Registry of the International Society for Heart and Lung Transplantation: Eleventh Official Pediatric Heart Transplantation Report: 2008. The Journal of Heart and Lung Transplantation, 27, 970-977. http://dx.doi.org/10.1016/j.healun.2008.06.016

[14] Pophal, S.G., Sigfusson, G. and Booth, K.L. (1999) Complications of Endomyocardial Biopsy in Children. Journal of the American College of Cardiology, 34, 2105-2110. http://dx.doi.org/10.1016/S0735-1097(99)00452-0

[15] Andrews, R.E., Fenton, M.J., Ridout, D.A. and Burch, M. (2008) New-Onset Heart Failure Due to Heart Muscle Disease in Childhood. Circulation, 117, 79-84. http://dx.doi.org/10.1161/CIRCULATIONAHA.106.671735

[16] Hsu, D.T. and Canter, C.E. (2010) Dilated Cardiomyopathy and Heart Failure in Children. Heart Failure Clinics, 6, 415-432. http://dx.doi.org/10.1016/j.hfc.2010.05.003

[17] Dipchand, A.I., Kirk, R., Mahle, W.T., Tresler, M.A., Naftel, D.C., Pahl, E., Miyamoto, S.D., Blume, E., Guleserian, K.J., White-Williams, C. and Kirklin, J.K. (2013) Ten yr of Pediatric Heart Transplantation: A Report from the Pediatric Heart Transplant Study. Pediatric Transplantation, 17, 99-111. http://dx.doi.org/10.1111/petr.12038

[18] Pietra, B.A., Kantor, P.F., Bartlett, H.L., Chin, C., Canter, C.E., Larsen, R.L., Edens, R.E., Colan, S.D., Towbin, J.A., Lipshultz, S.E., Kirklin, J.K., Naftel, D.C. and Hsu, D.T. (2012) Early Predictors of Survival to and After Heart Transplantation in Children With Dilated Cardiomyopathy, Circulation, 126, 1079-1086. 